\title{
Momordica charantia constituents and the anti-proliferative activity against an ovarian cancer cell-line SKOV-3
}

*Vishal Pundir ${ }^{1}$ and Aditya Chandel ${ }^{2}$

\author{
1 Department of Botany, \\ Tilakdhari Post Graduate College, \\ JAUNPUR, UTTAR PRADESH - 222002 INDIA \\ 2323 Dr. Martin Luther king Jr Blvd \\ New Jersey Institute of Technology, \\ NEWYORK, NJ 07102, U.S.A. \\ *Corresponding Author
} Received : 11.07.2021; Accepted : 10.08.2021

\begin{abstract}
Momordica charantia fruits contain terpenoidal saponins, phenolic acids, minerals and amino acids, all of which are shown to confer beneficial effects on human health. This study describes a method to conduct qualitative composition analysis of bioactive compounds in the fresh juice of Momordica charantia fruits harvested locally in Jaunpur (Uttar Pradesh, India) grown under traditional farming conditions. Fruit juice extract consisting primarily of phenolic acids, showed presence of 12 compounds. Crude extract inhibited the viability of a platinum resistant ovarian cancer cell-line SKOV-3 in-vitro during 48 hours of treatment.

\section{Introduction}

Epithelial Ovarian Cancer (EOC) being a highly asymptotic disease is a clinically challenging gynecologic malignancy $3,6,13,15$. Majority of EOC cases are diagnosed with disseminated stage III/IV disease. World-wide over 295,414 cases of EOC were identified in the year $2018^{3,6}$. In India alone, over 59,276 new EOC detections were expected to occur in the year 2020. It is anticipated that over 371,000 new cases will be diagnosed annually by the year 2035 followed by 254,000 deaths.

Debunking surgery combined with platinum/ paclitaxel chemotherapy generates nearly complete response initially ${ }^{13,15,20,21}$. Unfortunately, most of the EOC patients experience disease recurrence presenting lifethreatening broad-spectrum chemotherapy resistance disease which proves fatal ${ }^{13,15,20,21}$. Age and familial history of EOC and breast cancer constitutes the major risk factors ${ }^{3,6}$. Despite recent improvements in the understanding of EOC disease and introduction of novel therapies, overall five year survival rate of EOC patients remains below $40 \%{ }^{13,15,20,21}$. Identification of new targets and their modulators are warranted. In addition, identification of dietary supplements that can suppress driver signaling pathways in EOC and control EOC proliferation can improve survival rate of women at risk of EOC.

In this report, a method to process, isolate and analyze phytochemical constituents of Momordica charantia fruit using HPLC-ESI-MS-MS techniques; and the effects of crude constituents on the proliferation of SKOV-3, an ovarian cancer cell-line are described. Our analysis showed that over 12 characterized phenolic acids and hydrolysable tannins were present in Momordica charantia juice. Phytochemicals present in crude extracts of Momordica charantia reduce proliferation of platinum resistant EOC cell-line in-vitro.

\section{Materials and Methods \\ Momordica charantia fruits}

Momordica charantia, used in this study were grown in the normal soil conditions in Jaunpur. Fruits were harvested and stored under refrigeration in the Department of Botany, TDPG College Jaunpur till processing and 


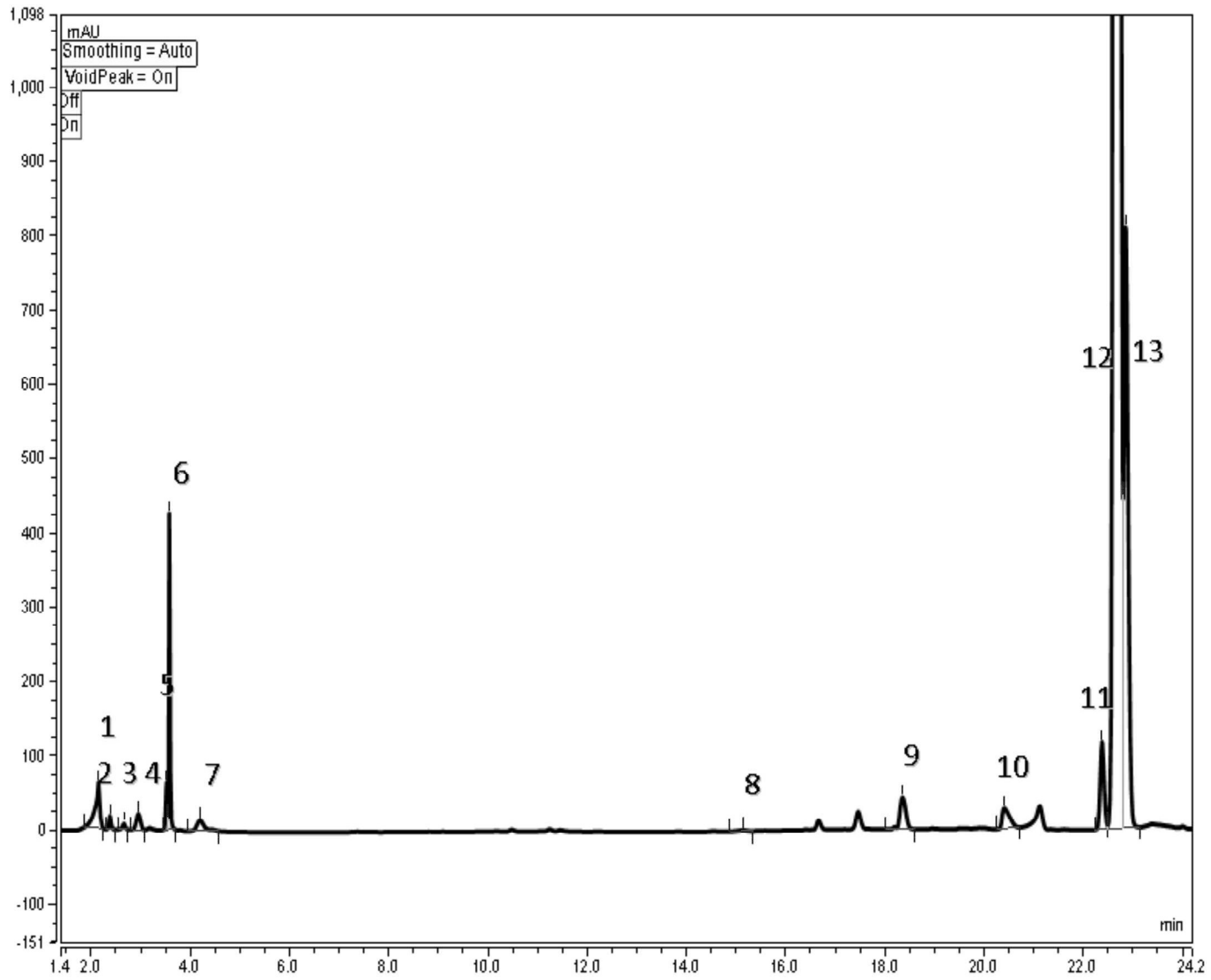

Fig, 1 : HPLC-PDA profile of Momordica charantia juice extract

analysis.

\section{Chemicals}

In-house phenolic standards were used. HPLC grade methanol, acetonitrile, formic acid and water were procured from Fisher Scientific (USA).

\section{Extraction of phenolic acids and flavonols}

The refrigerated fruits were ground using a commercially available coffee grinder, filtered using cloth. Sodium benzoate was added and extracts were dried in oven at $45^{\circ} \mathrm{C}$ for 48 hours.

\section{Analysis of phenolic acids and Tannins}

Dried juice extract was unthawed, re-dissolved in mobile phase (water, $1 \mathrm{~mL}$ ) and filtered using a PVDF syringe filter. The filtered extract was analyzed by HPLC and LC-MS-MS techniques. Phenolic acids and tannins were analyzed in Dionex UltiMate-3000 LC system.

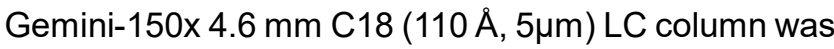

used in the analyses. Phenolic acid and tannins were detected at $280 \mathrm{~nm}$ wavelength. Column was equilibrated using $100 \%$ Solvent-A for 5 min before and after each injection. Flow rate was maintained at $1 \mathrm{~mL} / \mathrm{min}$. SolventA: Mobile phase (water $+0.5 \%$ formic acid). Solvent-B: Acetonitrile $+0.5 \%$ formic acid. Gradients: 0 min, $100 \%$ A (solvent-A: water+0.5\% formic acid); $10 \mathrm{~min}, 20 \% \mathrm{~A} ; 20$ min, $40 \% A$; 40 min, $0 \% A$; maintained at $A(0 \%)$ for 15 min. Effluents from the column was introduced into the triple-quadrupole mass spectrometer (ESI ion source).

\section{MS Spectrometry}

LC/MS-MS analyses were conducted using an Applied Biosystems API 3000TM triple-quad mass spectrometer coupled with Dionex UltiMate-3000 LC system. MS data were obtained in chemical ionization $(\mathrm{APCl})$ in -ve/+ve ion detection mode under atmospheric pressure. Curtain gas was set to $12 \mathrm{psi}$, nebulizer gas 
TABLE-1 : Retention times (RT) and area percentage of identified acids Momordica charantia juice extract. Details of instrumentation, methods of analyses and calculations are provided in Materials and Method sections.

\begin{tabular}{c|c|c|c}
\hline Peak no & Compound name & Retention time in min & Area in \% \\
\hline 1 & p-Coumaric acid & 2.146 & 1.07 \\
\hline 2 & Cinnamic acid & 2.377 & 0.12 \\
\hline 3 & Catechin & 2.66 & 0.08 \\
\hline 4 & Vicine & 2.951 & 0.3 \\
\hline 5 & Genistic acid & 3.575 & 1.28 \\
\hline 6 & Gallic acid & 4.198 & 0.32 \\
\hline 7 & Sinapic acid & 15.145 & 0.04 \\
\hline 8 & Vannilic acid & 18.355 & 0.79 \\
\hline 12 & Chlorogenic & 20.407 & 0.64 \\
\hline 10 & Syringic acid & 22.377 & 1.39 \\
\hline
\end{tabular}

pressure was set to $7 \mathrm{psi}$ ), nebulizer current was at $2.0 \mathrm{~mA}$ and entrance potential was set to $-10 \mathrm{~V}$. Focusing potential was at $-300 \mathrm{~V}$, declustering potential was set to $-60 \mathrm{~V}$, and collision energy was put at $-50 \mathrm{~V}$, whereas the collision cell exit potential was set to $-5.0 \mathrm{~V}$. The source temperature was set at $500{ }^{\circ} \mathrm{C}$. Compounds were identified by comparing retention times (RTs), UV spectral patterns, and APCl-MS-MS fragmentation patterns.

\section{Cell viability assay}

SKOV-3 EOC cells were seeded triplicate (5000/ well) in a round bottom 96-well cell culture plates in 100uL (10\% FBS)+1\%Strep supplemented DMEM medium and allowed to adhere and incubate overnight. Water (vehicle) or Momordica charantia dry powder dissolved in 100uL medium was added to the wells. The cells were incubated with vehicle/Momordica charantia extract solution for 48 hours. Media was replaced with MTS (Promega, Celltiter96Aqueous one solution, 1:10 catalog number G3580). The cells were incubated for 2-3 hours. The optical density (OD) of the cells was red by a spectrophotometer at 490nM wavelength. The \%age viability of Momordica charantia treated cells was calculated relative to water (vehicle) treated cells normalized to $100 \%$. Each datapoint was subtracted with average O.D. values of 3 cell free blank cells containing RPMI media and MTS dye.

\section{Statistical analysis}

The viability of the SKOV-3 cells in the treatment versus vehicle treated cells was compared by $t$-test using GraphPrism Pad $8^{\text {th }}$ edition.

\section{Results}

Momordica charantia is rich in polyphenolics and tannins: HPLC-ESI-MS-MS analyses showed that Momordica charantia crude extracts contained gallic acid, p-coumaric acid, genistic acid, sinapic acid, syringic acid, cinnamic acid, chlorogenic and catechin, vannilic acid, vicine along with benzoic acid and protocatechuic acid in varying compositions ${ }^{4}$. Protochatechuic acid was found 


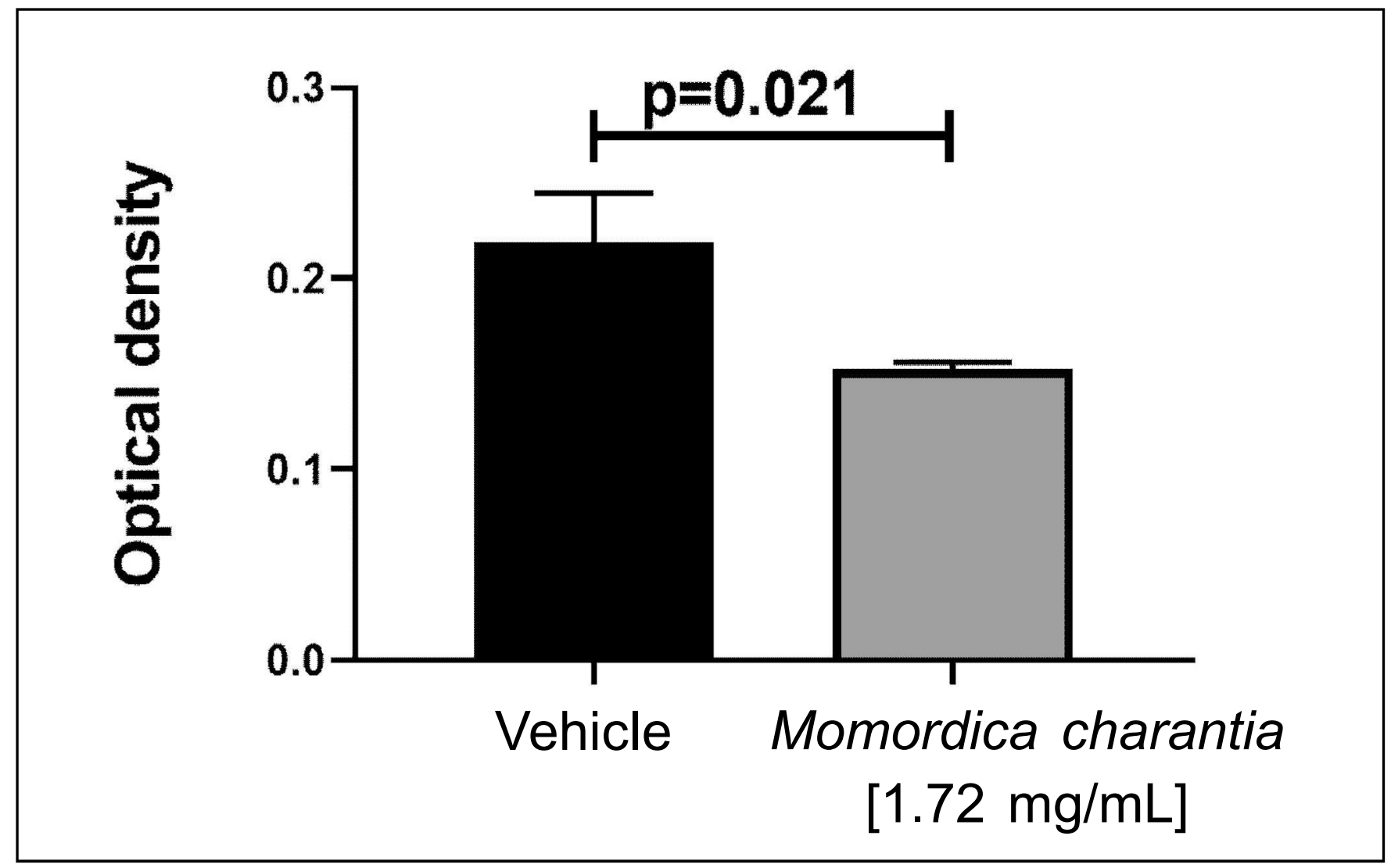

Fig. 2 : Momordica charantia juice extract reduced the viability of SKOV-3 ovarian cancer cells. The optical density values in treated group were lower than vehicle group (t-test, two tailed, $p=0.02$ ).

to be the most enriched compound in the crude extract. The LC-MS profiles and retention times (rt) of each of these constituents are shown in the Fig. 1 and Table- 1. Rest of the constituent were present in less than $1.5 \%$ each (Fig.1 and Table-1).

Momordica charantia extracts inhibited the viability of SKOV-3 ovarian cancer cells: Treatment with Momordica charantia crude extract inhibited the viability of SKOV-3 cells significantly (Fig. 2, $p=0.02$ ) within 48 hours of treatment.

\section{Discussions}

Momordica charantia belongs to the Cucurbitaceae family and is being increasingly consumed worldwide due to emerging in-vitro and in-vivo data demonstrating antioxidant, anti-inflammatory, anti-cancer, anti-diabetic, antibacterial, anti-obesity, and immunomodulatory activities $1,6,7,8,10,11,15$. Safety and efficacy of Momordica charantia in pre-diabetes and type 2 diabetes mellitus patients was shown ${ }^{2,16,17}$. The antibacterial effects of Momordica charantia against methicillin-resistant Staphylococcus aureus was also shown ${ }^{13}$. Momordica charantia extract showed hypoglycemic effect and reduced hepatic lipid accumulation in ob/ob mice ${ }^{9}$. Similarly, Momordica charantia polysaccharides was shown to reduce oxidative stress, inflammation, and apoptosis in ethanol-induced gastritis in mucosa through NF-kB inhibition ${ }^{21,22}$.

In our studies, Momordica charantia extracts showed significant inhibition of SKOV-3 cells, an ascites derived platinum resistant ovarian cancer cell-line. Which constituent of Momordica charantia is primarily responsible for anti-viability effects against SKOV-3 cell-lines remains unclear. Our future studies aim to purify and isolate each of the constituents in singular homogeneity to test and validate the anti-viability effects against platinum resistant ovarian cancer cell-line.

Ovarian cancer is a lethal malignancy that about one in every 75 women face ${ }^{3,6,12-14}$. Current approaches of surgical-debulking combined with chemotherapy or the newer advances in understanding of ovarian tumorigenesis and introduction of novel therapies do not rescue every EOC patients from the death $3,5,6,20$. About $40 \%$ patients still perish due to disease within 5 years. Women in developing countries like India face greater threats to their lives if diagnosed with ovarian cancer ${ }^{19}$. Prevailing poverty, environmental pollution, lack of education and womendedicated health care facilities combined with genderdiscriminatory cultural practices, predispose women in 
India considerably to the increased dangers of cancers and deaths including from EOC. Therapeutic benefits of dietary supplements and foods or fruits like Momordica charantia ${ }^{21}$ can significantly improve the outcomes of the women in India via dietary consumptions. Multi-layers of benefits exhibited of Momordica charantia summarized above combined with the anticancer activity exhibited in our experiment (Fig. 2) shows the preliminary potential to reduce the disproportionate burden of ovarian cancer diseases that women in India face. Future studies will attempt to isolate biological activity relevant components and interrogate their mechanism of molecular actions in ovarian cancer models in-vitro and in-vivo.

\section{References}

1. Al-Ishaq RK, Overy AJ, Büsselberg D. Phytochemicals and Gastrointestinal Cancer: Cellular Mechanisms and Effects to Change Cancer Progression. Biomolecules. 2020; 8 : 10(1):105.

2. Bacanli M, Dilsiz SA, Ba aran N, Ba aran AA. Effects of phytochemicals against diabetes. Adv Food Nutr Res. 2019;89:209-238.

3. Bray F, Ferlay J, Soerjomataram I, Siegel RL, Torre LA, Jemal A. Global cancer statistics 2018: GLOBOCAN estimates of incidence and mortality worldwide for 36 cancers in 185 countries. CA Cancer J Clin. 2018;68(6):394424.

4. Clifford MN. Chlorogenic acids and other cinnamates: nature, occurrence and dietary burden. Journal of the Science of Food and Agriculture. 1999;79(3):362-372.

5. Coburn S, Bray F, Sherman M, Trabert B. International patterns and trends in ovarian cancer incidence, overall and by histologic subtype. Int J Cancer. 2017; 140(11):2451-2460.

6. Dandawate PR, Subramaniam D, Padhye SB, Anant S. Bitter melon: a panacea for inflammation and cancer. Chin J Nat Med. 2016;14(2):81-100.

7. Dutt R, Garg V, Khatri N, Madan AK. Phytochemicals in Anticancer Drug Development. Anticancer Agents Med Chem. 2019;19(2):172-183.

8. Dwijayanti DR, Shimada T, Ishii T, Okuyama T, Ikeya Y, Mukai E, Nishizawa M. Bitter melon fruit extract has a hypoglycemic effect and reduces hepatic lipid accumulation in ob/ob mice. Phytother Res. 2020;34(6):13381346.

9. Fang EF, Froetscher L, Scheibye-Knudsen M, Bohr VA, Wong JH, Ng TB. Emerging Antitumor Activities of the Bitter Melon (Momordica charantia). Curr Protein Pept Sci. 2019; 20(3):296-301.

10. Jia S, Shen M, Zhang F, Xie J. RecentAdvances in Momordica charantia: Functional Components and Biological Activities. Int J Mol Sci. 2017;18(12):2555.

11. Kilcar AY, Yildiz O, Dogan T, Sulu E, Takan G, Muftuler FZB. The Effect of Bitter Melon (Momordica charantia) Extract on the Uptake of <sup>99m</sup>Tc Labeled Paclitaxel: In Vitro Monitoring in Breast Cancer Cells. Anticancer Agents Med Chem. 2020;20(12):1497-1503.

12. Kossaï M, LearyA, Scoazec JY, Genestie C. Ovarian Cancer: A Heterogeneous Disease. Pathobiology. 2018;85(12):41-49.

13. Kuok CF, Hoi SO, Hoi CF, Chan CH, Fong IH, Ngok CK, Meng LR, Fong P. Synergistic antibacterial effects of herbal extracts and antibiotics on methicillin-resistant Staphylococcus aureus: A computational and experimental study. Exp Biol Med (Maywood). 2017;242(7):731-743.

14. Narod S. Can advanced-stage ovarian cancer be cured? Nat Rev Clin Oncol. 2016; 13(4):255-61.

15. Nerurkar P, Ray RB. Bitter melon: antagonist to cancer. Pharm Res. 2010; 27(6):1049-53.

16. Peter EL, Deyno S, Mtewa A, Kasali FM, Nagendrappa PB, Sesaazi D, Tolo CU, Ogwang PE. Safety and 
efficacy of Momordica charantia in pre-diabetes and type 2 diabetes mellitus patients: a systematic review and meta-analysis protocol. Syst Rev. 2018;7(1):192.

17. Peter EL, Kasali FM, Deyno S, Mtewa A, Nagendrappa PB, Tolo CU, Ogwang PE, Sesaazi D.J Momordica charantia L. lowers elevated glycaemia in type 2 diabetes mellitus patients: Systematic review and meta-analysis. Ethnopharmacol. 2019; 231:311-324.

18. Raish M, Ahmad A, Ansari MA, Alkharfy KM, Aljenoobi FI, Jan BL, Al-Mohizea AM, Khan A, Ali N. Momordica charantia polysaccharides ameliorate oxidative stress, inflammation, and apoptosis in ethanol-induced gastritis in mucosa through NF-kB signaling pathway inhibition. Int J Biol Macromol. 2018;111:193-199.

19. Roett MA, Evans P. Ovarian cancer: an overview. Am Fam Physician. 2009;80(6):609-16.

20. Shabir S, Gill PK. Global scenario on ovarian cancer. Its dynamics, relative survival, treatment, and epidemiology. Adesh Univ J Med Sci Res 2020;2(1):17-25.

21. Wang S, LiZ, Yang G, Ho CT, Li S. Momordica charantia: a popular health-promoting vegetable with multifunctionality. Food Funct. 2017;8(5):1749-1762.

22. Zhu F, Du B, Xu B. Anti-inflammatory effects of phytochemicals from fruits, vegetables, and food legumes: A review. Crit Rev Food Sci Nutr. 2018;58(8):1260-1270. 